Original Research Paper

\title{
Combination of Backward and Forward Approaches for Future Prediction by Business Intelligence Tools
}

\author{
${ }^{1 *}$ Mohammad Najjartabar Bisheh, ${ }^{2}$ Abbas Raissi and ${ }^{3}$ Saeedeh Mokhtari \\ ${ }^{1}$ Department of Industrial and Manufacturing Systems Engineering, Kansas State University, Manhattan, Kansas, USA \\ ${ }^{2}$ Department of Industrial and Manufacturing Systems Engineering, Iran University of Science and Technology, Tehran, Iran \\ ${ }^{3}$ Faculty of Engineering, K. N. Toosi University of Technology, Tehran, Iran
}

\begin{abstract}
Article history
Received: $20-10-2020$

Revised: $12-12-2020$

Accepted: 15-01-2021

Corresponding Author: Mohammad Najjartabar Bisheh Department of Industrial and Manufacturing Systems Engineering, Kansas State University, Manhattan, Kansas, USA

Email: Mnajjartabar@ksu.edu
\end{abstract}

\begin{abstract}
Business intelligence tools now play an important role in data collection, processing and analyses of organizations. Managers may use outputs of such tools and make improved decisions. To provide an even better situation for managers, business intelligence tools may be used to predict future and display present and past statuses of the organization. Available business intelligence tools predict future based on historical data, i.e., they are said to enjoy backward approaches. In this study, it was tried to change extrapolation of historical trends according to our expectations of future events so that forward approaches could be employed for prediction purposes.
\end{abstract}

Keywords: Business Intelligence, Backward Prediction, Forward Prediction

\section{Introduction}

Business intelligence is the ability to collect, process and cumulate organizational information so that all staff in every level could access what they need, hence no competitive risk may be observed (Bahrami et al., 2012). Conventional tools are well capable of analyzing every aspect of the organization; however, all they process is historical data (events happened by now) which enables the organization to more accurately review its right or wrong decisions in the past. Yet, the past cannot be changed. Under these circumstances, managers need to predict future, control upcoming events and proceed in accordance with their goals. To make this possible, most manufacturers of business intelligence tools use predictive analyses to recognize variation patterns of indices and predict customers' future (Ranjan, 2009).

Future prediction of different parameters such as demand, price, failure rate, or even weights for strategies have been used in different context such as competition and game problems (Esmaeili et al., 2015; Mohamadi and Bahrini, 2020), complex systems (Majlesinasab et al., 2019a; 2019b; 2019c), supply chain management (Najjartabar-Bishehet al., 2018; Najjartabar et al., 2016; Soleimanynanadegany and Tolooie, 2015; Tolooie and Soleimanynanadegany, 2005; Tolooie et al., 2020; Zahraee et al., 2020), crowd sourcing and human computer interaction (Saremi et al., 2020a; 2020b;
Urbaczek et al., 2020), healthcare systems (Fanoodi et al., 2019; Jahantigh et al., 2017; Maleki et al., 2014; Malmir et al., 2016; Sepehri et al., 2013), quantitative strategy planning (Khanmohammadi et al., 2019; Montazeri and Najjartabar-Bisheh, 2017) and quality control (Daryabari et al., 2019). Two general types of models have been proposed for future prediction (Van den Poel and Buckinx, 2005). The first type, based on historical data, tries to recognize data variation patterns and extend it to predict the future. The second type models enjoy a qualitative approach according to which expert ideas are used for prediction. Each model has shown to have several advantages and defects. Quantitative models reflect clearly prior patterns and trends; however, they are incapable of considering events of no historical background. On the other hand, while qualitative models focus less on prior events (since they are thoroughly based on expert judgments) they may be used to estimate new events and their impact on predictions.

It is concluded that one may combine both quantitative and qualitative models and enjoy their advantages at the same time; besides, their defects may be solved when these models are used simultaneously. The present study suggests a method to combine these approaches. Quantitative techniques were used in the first phase and then the obtained outputs were subject to review through forward approaches and expert ideas. Therefore, inaccuracy of qualitative methods could be 
solved and quantitative techniques are no longer in the state of without unknowingness.

\section{Literature Review}

Many applications for business intelligence have been defined so far and their tools have been used in different categories (from both theoretical and practical points of view). Bahrami et al. (2012) established a proper procedure to employ business intelligence in market management. From his viewpoint, business intelligence capabilities are: Decision support, statistical analysis, future prediction and data analysis. Tutunea and Rus (2012) suggested use of business intelligence in Small and Medium Environments (SME). They believed that making right decisions at the proper time and consequently adopting proper procedures would be used as output of a business intelligence system creating competitive advantages for SMEs. Nofal and Yusof (2013) proposed a model to combine Enterprise Resource Planning (ERP) and business intelligence. They believed ERP may be used as a strategic tool for uniform planning and management of an organization. Moreover, business intelligence tools are well capable of supporting management decisions. Through combining these two models, one may enjoy their advantages simultaneously. It was claimed that this action improved data analysis and business trends predictions.

Foshay and Kuziemsky (2014) studied the feasibility of business intelligence in hygiene issues. Lasi (2013) provided a path to apply business intelligence in manufacturing environments. McBride (2014) carried out a case study on the use of business intelligence in a production chain. This study showed the importance of business intelligence in the management of the production chain. He expressed that applicability of business intelligence is not only for examination of current situation, but also for future prediction. He mentioned that by application of business intelligence in predictions through extrapolation of existing data, report making would no longer be their only use and the future may be revealed. Regression model is applied for prediction in McBride's method.

Chang (2014) introduced a new concept of business intelligence as business intelligence service in cloud environment. From his point of view, financial issues of an organization are caused by the lack of sufficient and accurate information regarding upcoming risks. Using business intelligence in cloud environment, one may take advantage of cloud calculus and complex models for modelling to increase accuracy in risk analysis and predictions. Chang developed a model to help users in reviewing their actions as well as predicting the future.

It is crystal clear that many applications for business intelligence have been defined and its tools have been used in various fields. Business intelligence tools have shown an undeniable role in providing information for future prediction. It would be meaningful to conclude that an essential use of these tools is future prediction or predictive analysis.

\section{How to Predict Future by Prevailing Business Intelligence Tools?}

Some dominant tools of business intelligence and their approach for prediction of future are discussed in this section. It is worth mentioning that all tools have strategies for predictive analysis; however, the prediction procedure may differ from one tool to another. In Tableau, future prediction is carried out via historical data. This software tries to find past-concerning data and predict future by extending them. Tableau has eight equations for data fitting and automatically selects an equation which is closer to the actual data at each prediction step so that the quality is improved. Besides, the user is given the opportunity to select the prediction equation manually (Tableau Software, 2019). Alteryx is another tool providing a platform to process a great deal of data in conjunction with predictive analysis. Future prediction is performed according to the historical data by means of the following prediction models: Regression, decision tree and the forest method.

Prediction by Qlik View is performed by What-if analysis. In other words, changing effects of independent variables on dependent ones are evaluated. Historical data are not sufficient in this tool and the user may predict independent variables according to the information they have in mind and estimate their effect on dependent variables (IBM, 2011). Microsoft provides users with What-if order (similar to Qlik); in addition, users may find out what changes are needed to be made to reach a certain goal through the Goal Seeking option.

Similarly, IBM tool predicts the future according to historical information. After collection and consolidation of data, future values are predicted by model fitting and determination of data change pattern. Decision tree algorithms, linear regression, kth average, ABC analysis and balanced score table are employed in SAS tool for predictions (SAS Institute Inc., 2015). Independent variables are predicted according to dependent ones and the prediction model is selected automatically based on data trends (Thompson and Duling, 2010). TIBCO Spotfire provides an environment for predictive analysis based on statistical methods. There is no need for statistical programming and the required output may be imported from other statistical tools such as SAS or MATLAB. Information Builder employs historical data in the prediction step and predicts the future by regression or decision tree.

Most business intelligence tools provide methods for future prediction; however, there have been few 
scientific studies on application of prediction in this field. Some prediction methods in prevailing tools were discussed so far. All existing tools of business intelligence predict future according to historical data. Actually, they try to find trends and patterns regarding the performance of an organization in the past and extend it to future periods. In the present work, authors attempted to develop a different approach by combining backward analyses with forward analyses.

\section{Prediction Model}

Two general types of models have been proposed for future prediction. The first type employs the knowledge of past and present; data variation patterns, processes, algorithms and change paths are determined and the future is predicted accordingly. Econometric techniques, trend lines (linear, exponential, polynomial, logarithmic, power, etc.) and time series (regression models, moving average, mixed moving average, etc.) are some of the methods applied in this category. These methods are sometimes called as without unknowingness due to the fact that merely historical data concerning past events are taken into account; in fact, they are incapable of assessing effects of new events in the future on existing trends. Quantitative models are never subject to be surprised, fairly inexpensive and highly inaccurate due to assuming the past continuing within the future.

On the other hand, the second type has a qualitative approach. It is believed that a given index in any scientific field depends on many factors which are neither readily recognizable nor follow a comprehensive model. On this ground, predictions based on quantitative methods may not yield acceptable answers for corresponding scientific fields (Taghva and Alizadeh, 2005). Human mind and knowledge about his surrounding environment are the basis of all proposed techniques for the second type of models. Panel, consensus, brainstorming, Delphi and scenario are some of the known techniques of this approach. Experts in a given field consider available historical data and suggest future events based on their understandings and personal ideas. It is necessary to mention that no accurate model is used in this approach, but intuition forms its structure which is known to be the main defect of the qualitative method (Jenkins and Smith, 1994). Plural thoughts are believed to choose a rather correct path; in other words, the more people suggest their ideas, the more reliable the output would be (Taghva and Alizadeh, 2005).

\section{Combination of Qualitative and Quantitative Approaches}

Each approach has been separately discussed so far and it was mentioned that they may have series of advantages and defects. Quantitative approaches vividly reflect past trends and patterns, while they are incapable of considering events with no historical background. On the other hand, qualitative methods are less accurate in reflecting past events (because they are only based on personal judgments); however, the effects of unprecedented events on future trends may be evaluated through such methods.

Therefore, one may combine qualitative and quantitative approaches and enjoy their advantages at the same time. In other words, simultaneous application of these approaches may cover their defects and faults. A procedure is hereby introduced through which these approaches may become one. Quantitative approaches were employed in the first phase and then the outputs were reviewed based on futuristic and expert ideas. Hence, inaccuracy of qualitative methods might be solved and quantitative approaches would no longer be without unknowingness. In other words, the proposed model helps to change extrapolation of historical trends according to existing expectations on future events. These events may include any type of action in technology, politics, society, economy and other affecting changes on trends. Phases of the proposed model are explained as follows.

\section{Phase I: Quantitative Prediction}

In the first step, trend lines (linear, exponential, polynomial, power, etc.) or time series (regression models, moving average, mixed moving average, etc.) of historical data were fitted and the closest model to the historical data was selected. Model selection strongly depends on the organization business and the nature of corresponding data. It is clear that there are so many methods in hand for this purpose while testing them all could be quite costly and time-consuming. Thus, several models, expected to show better fits according to the nature of organizational data, were selected (a primarily filtration) and then the best model among the selected models with the least difference from data points was determined.

\section{Phase II: Qualitative Prediction}

As mentioned before, qualitative methods are based on expert ideas, for collection and analyses of which, taking the following steps is necessary:

- $\quad$ First step: Appointing a supervisor to carry out the prediction process

- Second step: Determination of the number of individuals contributing to the prediction process

- Third step: Determination of a certain index to be predicted; there is no need for the organization to employ these models for prediction of all indices, but only important and decisive ones 
- Fourth step: Determination of a certain period for prediction; naturally, index value may vary within future days, months or years, then, a certain time is required to begin the prediction process

- $\quad$ Fifth step: Collecting individuals' ideas involved in the prediction process; authors accomplished this step inspiring from a common method in maintenance and repair industry called Failure Mode and Effects Analysis (FMEA). This method has been widely used in all fields of industry since 2000

\section{FMEA Method}

FMEA tries to find factors resulting in malfunction of machineries or equipment in future. Being a preventive method, FMEA helps to remove potential failures in products or process duration before they occur. There three major factors in FMEA to consider:

- Occurrence: Probability i.e., failure counts to process operation times ratio

- Severity: Evaluation of failure consequences (in case of occurrence). Severity is an evolution scale reflecting a failure in case of occurrence

- Detection: Probability of failure detection prior to observation of its consequences. Detection is the ability to find the reason and mechanism of failures

Probable failures and problems for a given instrument were initially listed. The three indices were given a value ranging from 1 to 10 for each problem. By multiplication of these indices, Risk Probability Number (RNP) is obtained:

\section{$R P N=$ Severity $\times$ Occurrence $\times$ Detection}

Priority in investigation goes to problems with higher RPN values since they are more likely to happen, hence, they would bring about harsh damages. An acceptable level of RPN has to be set in every FMEA project which is defined according to the organization. RPN $=125$ (Severity $\times$ Occurrence $\times$ Detection), for instance, could be an RPN level for a hypothetical FMEA project. Accordingly, RPN $>125$ events have to be prioritized for proper modifying procedures. Either Pareto diagram or zoned diagram may be used in order to determine cases with more priority.

\section{Extension of FMEA Model for Prediction in Business Intelligence Tools}

FMEA model was extended and employed for prediction. RPN method may be appropriate to present expert ideas on the prediction curve. As mentioned before, certain index and prediction period were given to individuals involved in the process. Each person suggested a list of probable events which in case of happening would cause some changes to quantitative predictions of that index. This step may be performed via any qualitative method such as consensus, Delphi, brainstorm, etc. According to these methods, people involved in the prediction process were to suggest a list of probable future events. However, past and present events affecting the studied index were also taken into consideration.

After preparation of the lists, they were sent to the prediction process supervisor. He integrated the ideas and removed similar ones. Then, the supervisor propounded each idea and questioned everyone regarding a certain event (which is proposed by one or more individuals). It is best to discuss each event separately so that people would not get involved in so many cases and their mutual influences. Similar to FMEA, experts were required to set a probability value for occurrence, severity and detection of a certain event. Consequences of failure and damage are always studied in FMEA (negative effects), while business intelligence (the proposed model) identifies opportunities and threats for an organization (Vinekar et al., 2009). Hence, in order to use FMEA method in business intelligence tools, one has to consider all the events (imposing both positive and negative events). The individuals filled out a form designed in this system according to their understandings (Table 1). The form header shows the given index and prediction time period. For each event, there was one form to be filled.

Severity, Occurrence and detection are given the corresponding values in FMEA method. These concepts may be redefined in business intelligence predictions as follows:

1. Severity: In case of occurrence of an event, how effectively it alters the index and quantitative prediction

2. Occurrence: How probable the event is or in case of occurrence, how probable its effectiveness is

3. Detection: To what extend it is possible to detect the consequences; in other words, how clear the consequences of probable events are

Table 1: The proposed form to collect expert ideas in prediction process

\begin{tabular}{|c|c|c|c|c|c|}
\hline \multicolumn{2}{|l|}{ Predictor's name } & \multicolumn{2}{|c|}{ Prediction index } & \multicolumn{2}{|c|}{ Prediction period } \\
\hline Predicted event & Severity & Occurrence & Detection & Effect bias & Priority number \\
\hline
\end{tabular}


Users might assign a score ranging from 1 to 10 to the three indices (higher values imply higher chance of occurrence, more severity, or easier detection). In addition to the three indices, the effectiveness of an event is also necessary to be realized. Effectiveness illustrates negative or positive influence of an event on the studied index (it consists of two positive and negative characters). Effectiveness is not used in FMEA due to the fact that all events investigated in that area have negative consequences and cause damage, while in business intelligence, different events may lead to either positive or negative actions. By multiplication of the three indices in FMEA, Risk Priority Number (RPN) may be obtained. However, the multiplication result in the proposed model was named PN because of simultaneous consideration of positive and negative events. PN values were automatically calculated by the system which equal occurrence $\times$ severity $\times$ effect bias (the detection effect may be considered differently that is discussed in the following sections).

\section{A Method to Achieve Consensus in Expert Ideas}

It was already mentioned that contribution of more individuals in the prediction process leads to more reliable results. Hence, it is recommended that the prediction process is carried out through a team work. Under these circumstances, the displayed form in Table 1 is filled out by every individual in the team. In the meantime, another method is needed to integrate ideas i.e., all ideas have to be summarized and the outcome is illustrated in the prediction result. Importance of each individual in the team has to be manifested by a weight factor determined by the senior manager; there are two probable situations:

1. The senior manager thinks that ideas of all individuals are equally important. In this case, knowing the number of contributors, the weight factor of each individual may be obtained by the following formula:

\section{Individual's weight factor \\ $=1 /$ Number of contributors}

2. The senior manager thinks that ideas are of different importance and some ideas are preferred to others. In this case, the senior manager is required to assign a weight factor $(<1)$ to contributors so that the total value is 1

After determining the weight factor, the ideas have to be integrated. A PN value is calculated for each individual and event; then, all $\mathrm{PN}$ values have to be integrated, carried out when they are sent to the supervisor using the following formula.

It was already mentioned that contribution of more individuals in the prediction process leads to more reliable results. Hence, it is recommended that the prediction process is carried out through a team work. Under these circumstances, the displayed form in Table 1 is filled out by every individual in the team. In the meantime, another method is needed to integrate ideas i.e., all ideas have to be summarized and the outcome is illustrated in the prediction result. Importance of each individual in the team has to be manifested by a weight factor determined by the senior manager; there are two probable situations:

1. The senior manager thinks that ideas of all individuals are equally important. In this case, knowing the number of contributors, the weight factor of each individual may be obtained by the following formula:

\section{Individual's weight factor $=1 /$ Number of contributors}

2. The senior manager thinks that ideas are of different importance and some ideas are preferred to others. In this case, the senior manager is required to assign a weight factor $(<1)$ to contributors so that the total value is 1

After determining the weight factor, the ideas have to be integrated. A $P N$ value is calculated for each individual and event; then, all $P N$ values have to be integrated, carried out when they are sent to the supervisor using the following formula:

$$
P N_{y}=\sum_{x=1}^{N} W_{x} P N_{x y}, y=1,2, \ldots, M
$$

\section{Phase III: Combination of qualitative and quantitative methods}

Previous phases yield a prediction curve by quantitative methods and a list of probable events with corresponding PN values. These data are now integrated and the effect of future events is illustrated on the prediction curve. On this purpose, the events are divided into two categories:

1. Historical data on a predicted event are available, for example:

a. According to a governmental order, consumption of several products is reduced (or increased)

b. A client becomes bankrupt and ceases his purchase 
c. A client is expanding his work; thus, he would increase his demand

d. Due to some policies, the organization decides to shut down a store

2. Historical data about a predicted event are not available; for example:

a. The organization introduces a new product

b. A new client is registered

c. The organization opens a new store

d. A new competitor enters the market

e. A competitor loses his share in the market because of a destructive fire

Prediction of effectiveness is carried out readily in case of events in the first category. Historical information about products, clients, stores, etc. is available and the effectiveness is accordingly determined. Let us take reduction in sale of a specific product (due to a governmental order, increase in costs of raw materials, change of buyers' taste, etc.) as an example. Historical sale data of this product are available. It is assumed that $10 \%$ of the total sale of the organization was due to this product. As mentioned in the previous section, individuals assign values of occurrence, severity and detection. In this example, contributors answer the following questions:

1. What is the probability of occurrence? Answer: 8

2. In case of occurrence, what would happen to the sale of this product? (would it be totally lost or there would be some increases or decreases?) Answer: 6

3. How clear are the consequences? Answer: 4

4. Does the event affect the studied index positively or negatively? Answer: positive

Now, it is required to illustrate the effect on the prediction curve. At first, RPN is calculated:

$$
R P N=\text { effect bias } \times \text { severity } \times \text { occurrence }=0.6 \times 0.8=0.48
$$

Therefore, the Sale of this product, quantitatively predicted to be 1000 , increased by 1048 . There are many complexities regarding the prediction of second-category events due to the absence of corresponding historical data. In this situation, users are required to determine share of events. When the organization is planning to introduce a new product, for example, it has to illuminate its share in the market, thus, the effect may be shown on the prediction curve.

\section{Using the Detection Measure of Occurred Events}

So far, PN was realized as multiplication of effectiveness probability of an event by its effect order on the studied index. However, the detection of effects was valued by contributors in FMEA method. Occurrence and severity show the probability and effectiveness of a certain event, while the third index implies how easily these effects are recognized. The difference of indices is explained through an example: One predicts Iran-Korea game 2-0. The same person predicts Iran-Angola game 2-0 as well. The probabilities of these results as well as their effectiveness on Iran scores were equal for the predictor. However, the prediction of Iran-Korea game is more difficult because it depends on many factors. So, the probability index or easiness of detection of consequences may be used.

This index shows that whether the predictor clearly observes the event and can estimate its consequences or not. The value of this index is illustrated on the prediction curve by means of another method (1 to 10 values are given to the index; the higher the index, the easier detection of consequences). A main goal of business intelligence tools is to be simply presented so that users may realize the conditions in a short glance. Hence, the detection index is presented so that the user may readily comprehend the consequences.

In order to present the detection index, its value for each event was initially multiplied by the individuals' weight factor and then the total value was calculated. Then, the average value of the index for every event was obtained. A colored spectrum was used to present events on the prediction curve. The individuals had predicted several events according to their thoughts and determined their corresponding effectiveness. However, the prediction of consequences is not entirely simple (detection index is somehow close to 1). In such a case, the prediction curve is illustrated in a pale mode. On the other hand, in case of consensus among contributors on simplicity of estimation of consequences for people, the prediction curve is illustrated in a colorful mode.

Accordingly, when an index is predicted to decrease and the curve is colorful, one may conclude that the studied event is likely to happen and lead to significant consequences, thus, the user has to look for some compromises. But if the diagram is pale, there will be a chance for the index value decrease; though, the consequences will not be vivid.

\section{Conclusion}

In the present work, a model simultaneously applied quantitative and qualitative methods in prediction process of business intelligence tools. Quantitative methods properly reflect previous trends and patterns, yet, they are incapable of considering events of no historical background. Qualitative approaches, on the other hand, pay less attention to previous events (due to 
the fact that they are only based on judgments); however, they may consider new events and assess their consequences. Combining these two methods, one may enjoy their advantages at the same time.

The proposed model suggests predicting future via quantitative approaches and then, modifying the results according to expert ideas. Therefore, events of no historical background may be presented on the prediction curve and the prediction could be considered more close to the reality. Thus, the user of the business intelligence tool may observe variation trends of a certain index within time. Besides, applying quantitative approaches, they may predict future values of the index. Finally, if necessary, prediction may also be carried out through a qualitative procedure and the integrated results may be presented. Machine Learning (ML) models are taking over business prediction these days. However, ML model are still in the category of backward prediction. Combing forward prediction with ML model could be a good idea for future study of this paper.

\section{Author's Contributions}

All authors equally contributed in this study.

\section{Ethics}

This article is original and contains unpublished material. The corresponding author confirms that all of the other authors have read and approved the manuscript and no ethical issues involved

\section{References}

Bahrami, M., Arabzad, S. M., \& Ghorbani, M. (2012). Innovation in market management by utilizing business intelligence: introducing proposed framework. Procedia-Social and Behavioral Sciences, 41, 160-167.

Chang, V. (2014). The business intelligence as a service in the cloud. Future Generation Computer Systems, 37, 512-534.

Daryabari, S. A., Malmir, B., \& Amiri, A. (2019). Monitoring Bernoulli processes considering measurement errors and learning effect. Quality and Reliability Engineering International, 35(4), 1129-1143.

Esmaeili, M., Bahrini, A., \& Shayanrad, S. (2015). Using game theory approach to interpret stable policies for Iran's oil and gas common resources conflicts with Iraq and Qatar. Journal of Industrial Engineering International, 11(4), 543-554.

Fanoodi, B., Malmir, B., \& Jahantigh, F. F. (2019). Reducing demand uncertainty in the platelet supply chain through artificial neural networks and ARIMA models. Computers in biology and medicine, 113, 103415 .
Foshay, N., \& Kuziemsky, C. (2014). Towards an implementation framework for business intelligence in healthcare. International Journal of Information Management, 34(1), 20-27.

IBM. (2011). QlikView for Hyperion. IBM. https://www.ibm.com/support/pages/node/493031

Jahantigh, F. F., Malmir, B., \& Avilaq, B. A. (2017). A computer-aided diagnostic system for kidney disease. Kidney research and clinical practice, 36(1), 29.

Jenkins, D. A., \& Smith, T. E. (1994). Applying Delphi methodology in family therapy research. Contemporary Family Therapy, 16(5), 411-430.

Khanmohammadi, E., Malmir, B., Safari, H., \& Zandieh, M. (2019). A new approach to strategic objectives ranking based on fuzzy logarithmic least squares method and fuzzy similarity technique. Operations Research Perspectives, 6, 100122.

Lasi, H. (2013). Industrial intelligence-a business intelligence-based approach to enhance manufacturing engineering in industrial companies. Procedia CIRP, 12, 384-389.

Majlesinasab, N., Yousefian, F., \& Feizollahi, M. J. (2019a, July). A first-order method for monotone stochastic variational inequalities on semidefinite matrix spaces. In 2019 American Control Conference (ACC) (pp. 169-174). IEEE.

Majlesinasab, N., Yousefian, F., \& Feizollahi, M. J. (2019b). First-order Methods with Convergence Rates for Multi-agent Systems on Semidefinite Matrix Spaces. arXiv preprint arXiv:1902.05900.

Majlesinasab, N., Yousefian, F., \& Pourhabib, A. (2019c). Self-tuned mirror descent schemes for smooth and nonsmooth high-dimensional stochastic optimization. IEEE Transactions on Automatic Control, 64(10), 4377-4384.

Maleki, M., Majlesinasab, N., \& Sepehri, M. M. (2014). Two new models for redeployment of ambulances. Computers \& Industrial Engineering, 78, 271-284.

Malmir, B., Dehghani, S., Jahantigh, F. F., \& Najjartabar, M. (2016, June). A new model for supply chain quality management of hospital medical equipment through game theory. In Proceedings of the 6th international conference on information systems, logistics and supply chain, ILS 2016.

McBride, N. (2014). Business intelligence in magazine distribution. International Journal of Information Management, 34(1), 58-62.

Mohamadi, M., \& Bahrini, A. (2020). A NashStackelberg equilibrium model for internet and network service providers in the demand market-a scenario-based approach. Wireless Networks, 26(1), 449-461. 
Montazeri, M. M., \& Najjartabar-Bisheh, M. (2017) Optimizing technology selection for power smart grid systems: A case study of Iran Power Distribution Industry (IPDI). Technology and Economics of Smart Grids and Sustainable Energy, 2(1), 6.

Najjartabar, M., Shetaban, S., \& Malmir, B. (2016). An Integrated locationinventory model for supply chain network with correlated demand. In Proceedings of the 2016 international conference on information systems, logistics and supply chain, June (pp. 1-4).

Najjartabar-Bisheh, M., Delavari, M., \& Malmir, B. (2018). Role of third-party companies in a sustainable supply chain design. International Journal of Logistics Systems and Management, 30(1), 95-112.

Nofal, M. I., \& Yusof, Z. M. (2013). Integration of business intelligence and enterprise resource planning within organizations. Procedia technology, 11(2013), 658-665.

Ranjan, J. (2009). Business intelligence: Concepts, components, techniques and benefits. Journal of Theoretical and Applied Information Technology, 9(1), 60-70.

Saremi, M. L., Saremi, R., \& Martinez-Mejorado, D. (2020a). How Much Should I Pay? An Empirical Analysis on Monetary Prize in TopCoder. arXiv preprint arXiv:2004.12504.

Saremi, R., Saremi, M. L., Desai, P., \& Anzalone, R. (2020b). Is this the right time to post my task? an empirical analysis on a task similarity arrival in topcoder. arXiv preprint arXiv:2004.12501.

SAS Institute Inc. (2015). Analytics on the Front Line. SAS. https://www.sas.com

Sepehri, M. M., Maleki, M., \& Majlesi Nasab, N. (2013). Designing a redeployment model for located ambulances. International Journal of Industrial Engineering \& Production Management, 24(2), 171-182.

Soleimanynanadegany, A., \& Tolooie, A. (2015). Review on Cross Docking Quantitative Approaches. Journal of information systems research and innovation, 23-30.
Tableau Software. (2019). Tableau Blueprint. https://help.tableau.com/current/offline/enus/tableau_blueprint.pdf

Taghva, M. R., \& Alizadeh, Y. (2005). Oil Price Forecasting, Using Trend Impact Analysis (TIA) Technique. Industrial Management Studies, 3(11), 1-35.

Thompson, W., \& Duling, D. (2010). Rapid Predictive Modeling for Customer Intelligence. In Proceedings of the SAS Global Forum 2010 Conference.

Tolooie, A., \& Soleimanynanadegany, A. (2011). Review on role of knowledge management in supply chain management. Journal of Information Systems Research and Innovation, 17-22.

Tolooie, A., Maity, M., \& Sinha, A. K. (2020). A twostage stochastic mixed-integer program for reliable supply chain network design under uncertain disruptions and demand. Computers \& Industrial Engineering, 148, 106722.

Tutunea, M. F., \& Rus, R. V. (2012). Business intelligence solutions for SME's. Procedia economics and finance, 3, 865-870.

Urbaczek, J., Saremi, R., Saremi, M. L., \& Togelius, J. (2020). Scheduling Tasks for Software Crowdsourcing Platforms to Reduce Task Failure. arXiv preprint arXiv:2006.01048.

Van den Poel, D., \& Buckinx, W. (2005). Predicting online-purchasing behaviour. European journal of operational research, 166(2), 557-575.

Vinekar, V., Teng, J. T., \& Chennamaneni, A. (2009). The interaction of business intelligence and knowledge management in organizational decisionmaking. Journal of International Technology and Information Management, 18(2), 143.

Zahraee, S. M., Mokhtar, A. A., Tolooie, A., \& Asri, N. A. M. (2020). Performance evaluation of EFB biomass supply chain for electricity power generation based on computer simulation: Malaysia case study. In Advances in Material Sciences and Engineering (pp. 363-375). Springer, Singapore. 